\title{
Transcriptome analysis of rubber biosynthesis in guayule (Parthenium argentatum gray)
}

\author{
Solomon H. Stonebloom ${ }^{1}$ and Henrik Vibe Scheller ${ }^{1,2^{*}}$ (D)
}

\begin{abstract}
Background: Natural rubber is currently produced nearly exclusively from latex of the Para rubber tree, Hevea brasiliensis. The desire to reduce the environmental cost of rubber production, fears of pathogen susceptibility in clonal Hevea plantations, volatility in the price of natural rubber, and increasing labor costs have motivated efforts to diversify the supply of natural rubber by developing alternative rubber crops such as guayule (Parthenium argentatum Gray). In Hevea, latex is produced as an exudate following wounding while in guayule, rubber is deposited within the cortical parenchyma and its production is strongly influenced by environmental conditions.

Results: To better understand the enzymology and regulation of guayule rubber biosynthesis and to identify genes with potential uses in the improvement of rubber yields, we conducted de novo transcriptome assembly and differential gene expression analyses of this process in guayule. This analysis supports a role for rubber in the defense against pathogens, identified new enzymes potentially involved in the biosynthesis of rubber as well as transcription factors specifically expressed in rubber-producing tissues.

Conclusions: Data presented here will be useful in the improvement of guayule as an alternative source of natural rubber and in better understanding the biosynthesis of this critical polymer. In particular, some of the candidate transcription factors are likely to control the rubber biosynthesis pathway and are good targets for molecular breeding or engineering of guayule plants with higher and more consistent production of rubber.
\end{abstract}

Keywords: Parthenium argentatum, Guayule, Rubber biosynthesis, Transcriptome, Gene expression

\section{Background}

Natural rubber is a critical commodity currently produced from the latex of the Para rubber tree, Hevea brasiliensis. Global annual natural rubber production exceeds 13 million metric tons [1]. The demand for natural rubber has grown due to economic development and increased automobile ownership in Asia. This increased demand for rubber, in addition to competition for land with more profitable oil palm cultivation is a driver of deforestation in southeast Asia and threatens biodiversity [2]. The desire to reduce the environmental cost of rubber production, fears of pathogen susceptibility in clonal Hevea plantations, volatility in the price of

\footnotetext{
* Correspondence: hscheller@|bl.gov

1Joint BioEnergy Institute and Environmental Genomics and Systems Biology Division, Lawrence Berkeley National Laboratory, Berkeley, CA 94720, USA

2Department of Plant and Microbial Biology, University of California Berkeley, Berkeley, CA 94720, USA
}

(c) The Author(s). 2019 Open Access This article is distributed under the terms of the Creative Commons Attribution 4.0 International License (http://creativecommons.org/licenses/by/4.0/), which permits unrestricted use, distribution, and reproduction in any medium, provided you give appropriate credit to the original author(s) and the source, provide a link to the Creative Commons license, and indicate if changes were made. The Creative Commons Public Domain Dedication waiver (http://creativecommons.org/publicdomain/zero/1.0/) applies to the data made available in this article, unless otherwise stated.

natural rubber on the commodities market, and increasing labor costs for Hevea rubber production motivate efforts to diversify the supply of natural rubber by developing alternative crops such as guayule (Parthenium argentatum Gray) and Russian dandelion (Taraxacum kok-saghyz). Guayule is a xerophytic perennial shrub in the Asteraceae that produces high quality natural rubber, was widely studied in the United States during World War II as part of the Emergency Rubber Project and is currently being developed as an alternative rubber crop.

Guayule has major advantages over Hevea for the production of rubber in that it can be cultivated broadly across temperate and arid climates. Guayule produces high molecular weight natural rubber suitable for use in the manufacture of tires. Harvesting of biomass and rubber extraction are easily mechanized, unlike the labor-intensive tapping and latex collection required for 
Hevea rubber production. In guayule, rubber is deposited throughout the stem, primarily in the cortical parenchyma. As rubber is deposited intracellularly and not in an exudate, to harvest rubber whole guayule plants are mechanically harvested, baled and then processed to chemically extract rubber and other co-products. Furthermore, guayule latex does not elicit an allergic response in individuals allergic to Hevea latex and extended exposure to guayule latex does not produce an allergic response [3]. Unfortunately, breeding and true domestication of guayule are complicated by its polyploidy and facultative apomictic reproduction by which approximately $90 \%$ of seeds are not the product of sexual reproduction. Naturally occurring stands and breeding populations of guayule contain individuals with ploidy levels ranging from $2 \mathrm{n}=2 \mathrm{x}=36$ (diploid) to $2 \mathrm{n}=5 \mathrm{x}=90$ (pentaploid) [4, 5]. Progeny of individual guayule plants frequently exhibit aneuploidy and while diploid lines exist and are currently being used in breeding programs, diploids lack vigor and have reduced biomass and rubber yields, suggesting that tetraploid lines will be necessary for cultivars entering production [4].

Unlike Hevea rubber production where latex exudate is produced following wounding, rubber biosynthesis in guayule occurs in cells lining the resin ducts and in the cortical parenchyma and is strongly influenced by environmental conditions $[6,7]$. Rubber is made only when plants experience cold temperatures during the winter [8-10]. Several studies have characterized changes in the expression of rubber-producing genes and the activity of rubber biosynthetic enzymes following exposure of the plant to low temperatures either in the field or under controlled conditions [11, 12]. This climactic regulation of rubber biosynthesis limits the productivity of guayule plantations as rubber is produced exclusively during cold winter months. This also affects where guayule may be profitably cultivated outside of the high-desert environment of guayule's native range. Given that there is evidence for the transcriptional regulation of rubber biosynthesis and as traditional breeding approaches to increasing the rubber content of guayule have been challenging, here we aim to identify master regulator transcription factors regulating rubber biosynthesis in guayule to enable engineering of improved lines. We hope that ectopic expression of these transcription factors throughout the year will induce constitutive rubber production, increase rubber yield and potentially expand the productive range and economic viability of this alternative rubber crop. Furthermore, differential gene expression analysis of rubber producing and control tissues will elucidate metabolic processes occurring during rubber biosynthesis and help to identify additional enzymes involved in the biosynthesis of rubber.
To this end we established conditions inductive of rubber biosynthesis in guayule and validated the induction of rubber biosynthesis by extraction and qRT-PCR of known rubber biosynthetic genes. We then conducted an RNA-seq analysis of gene expression in rubber biosynthetic and control tissues, assembled the transcriptome and performed differential gene expression analyses. As rubber is produced specifically in the stems of plants exposed to low temperatures, sequencing libraries were prepared from stem and leaf tissues of cold-treated and control plants. Genes previously suspected to be involved in rubber biosynthesis were highly expressed in rubber biosynthetic tissues. We identified a set of genes encoding transcription factors and DNA-binding proteins that were specifically expressed in rubber-producing tissue as candidate regulators of rubber biosynthesis and that may be useful for the improvement of guayule rubber yields. Analysis of gene expression in rubber-producing tissues also informs upon the biological function of rubber production and the identification of new enzymes involved in rubber biosynthesis.

\section{Results}

\section{Induction of rubber biosynthesis}

Rubber biosynthesis in guayule occurs when the shrub experiences low nighttime temperatures below $5-7^{\circ} \mathrm{C}$ during the winter in the cortical parenchyma of stems [9-11, 13, 14]. Artificial exposure to low temperatures induces rubber accumulation in guayule in plants older than approximately 120 days [8]. Exposure to low, non-freezing night temperatures in the lab induces rubber biosynthetic activity in guayule [15]. The enzymatic rates of 3-hydroxy-3-methylglutaryl-CoA reductase and rubber transferase enzymes are also seasonally induced [16]. To study changes in gene expression associated with the production of rubber we sought to establish inductive and repressive growth conditions for guayule rubber biosynthesis in the lab and to validate that changes in the expression of genes associated with rubber biosynthesis were induced.

We simulated approximate summer and winter temperature regimes in guayule's native environment with parameters in concordance with previous studies of the induction of rubber biosynthesis in guayule $[11,12]$. While low temperature is likely the most important environmental factor for inducing rubber biosynthesis, we also changed daylength and day night temperature differential to simulate seasonal changes where guayule is grown. Summer conditions were simulated in growth chambers with a 16 -h day with $25^{\circ} \mathrm{C}$ daytime and $15^{\circ} \mathrm{C}$ nighttime temperatures and winter conditions with an 11 -h day with $25^{\circ} \mathrm{C}$ daytime and $5{ }^{\circ} \mathrm{C}$ nighttime temperatures. To validate the induction of rubber biosynthesis 
we extracted rubber particles from guayule plants exposed to simulated winter and summer conditions for six weeks (Fig. 1a, b). Large amounts of rubber were present in cold-induced plants, forming a thick mat of congealed rubber following centrifugation. Little rubber was present in plants grown in simulated summer conditions. The expression levels of genes thought to be involved in the biosynthesis of rubber, AOS (Allene Oxide Synthase) and CPT (Cis Prenyl-Transferase), have been shown to be weakly correlated with the induction of rubber biosynthesis by winter conditions in guayule [12]. To validate that these genes were induced in our rubber-producing plants we tested the relative expression of previously identified genes thought to be involved in guayule rubber biosynthesis in stems of plants exposed to simulated summer and winter conditions using real-time RT-PCR. As expected, significant up-regulation of AOS, CPT and FPS (farnesyl diphosphate synthase) transcripts was detected in plants grown

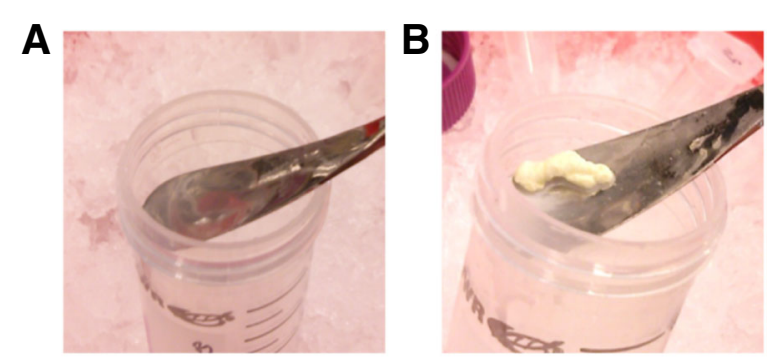

C

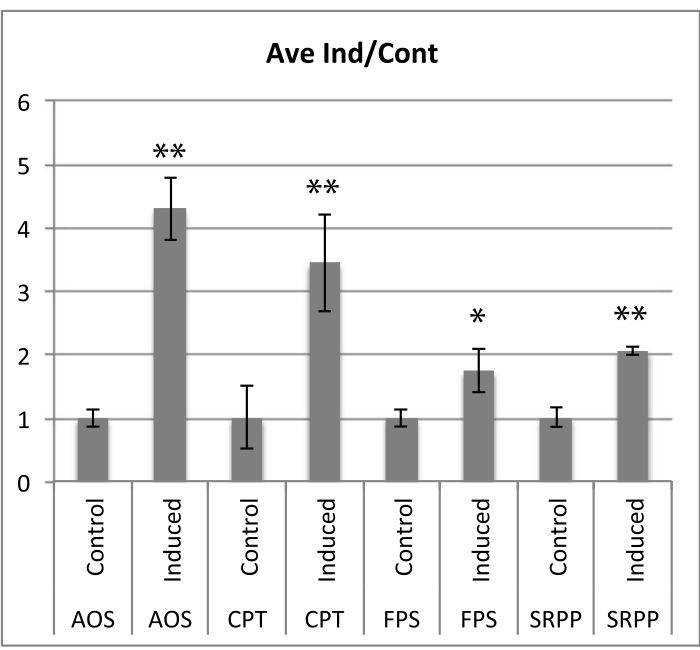

Fig. 1 Verification of rubber biosynthesis induction in guayule plants by exposure to simulated winter conditions for 2 months. Washed rubber particle extractions from plants grown in simulated summer (a) and winter (b) conditions. c Quantitative RT-PCR of rubber biosynthetic enzymes (AOS, allene oxide synthase; CPT, cis prenyl transferase; FPS, farnesyl diphosphate synthase; SRPP, small rubber particle protein). Significant differences between induced and control are shown (t-test, ${ }^{*} p<0.01,{ }^{* *} p<0.001$ ) in simulated winter conditions (Fig. 1c). We also detected significant induction of the SRPP (small rubber particle protein) transcript, which has not previously been shown to be responsive to low temperatures. We thus concluded that simulated winter conditions were capable of inducing rubber biosynthesis in guayule and that this induction elicited changes in the expression of rubber biosynthetic enzymes.

\section{RNAseq analysis and transcriptome assembly}

To characterize global changes in gene expression in rubber-producing tissues and to identify genes involved in the biosynthesis of rubber we performed an RNAseq analysis of control tissues (leaves) and tissues producing rubber (stems) from control and induced plants. We prepared bar-coded, directional RNA-seq libraries from the stems and leaves of 7-month-old guayule plants that had been exposed in simulated winter or summer conditions for 8 weeks (Additional file 1: Figure S1). Three biological replicate sequencing libraries were prepared from each tissue. These libraries were pooled and sequenced on MiSeq and Hiseq Illumina sequencers resulting in 28 million 300-bp paired-end reads and 155 million 150-bp paired-end reads respectively with an average of 2.4 million MiSeq and 12.9 million HiSeq reads per sample. The transcriptome was assembled using the Trinity assembler into 200,074 unigenes with a median length of $456 \mathrm{bp}$ and with $50 \%$ of the total sequence assembled into contigs of $850 \mathrm{bp}$ or larger (Table 1). The total assembly represented 149 million bp of sequence. As the plants used in this study are of the AZ-2, tetraploid cultivar, the transcriptome assembly contains up to four alleles of each gene given that the plants sequenced were the progeny of a tetraploid single plant selection [5]. Many transcripts were assembled into distinct "isoforms" likely representing distinct alleles of individual genes. That the transcriptome assembly likely represents up to four distinct contigs of each gene

Table 1 Summary statistics of contig assembly

\begin{tabular}{lc}
\hline Trinity 'unigenes' & 200,074 \\
\hline Contigs & 219,958 \\
GC content & $39.90 \%$ \\
Contig N10 & 2655 \\
Contig N20 & 1900 \\
Contig N30 & 1451 \\
Contig N40 & 1110 \\
Contig N50 & 850 \\
Median contig length & 456 \\
Average contig length & 678.1 \\
Total assembled bases & $149,145,074$ \\
\hline
\end{tabular}


complicates the data by adding allelic variation between individual plants to differential gene expression analysis. In the absence of a high-quality genomic sequence, it is generally not possible to distinguish between allelic variants and gene homologs. Furthermore, due to the relatively short contig length, it is obvious that several individual 'unigenes' can derive from the same gene locus.

\section{Differential gene expression analysis}

To identify genes involved in guayule rubber biosynthesis, differential gene expression analysis was conducted using the EdgeR package [17]. Our premise was that genes involved in rubber biosynthesis would be induced, primarily by cold, under simulated winter conditions in stems, where rubber is produced, but not in leaves where it is not; whereas genes induced by cold but not related to rubber biosynthesis would be induced in both leaves and stems. 16,394 differentially expressed contigs were identified at $p_{\text {adj }}<0.0001$ between two or more tissues. In a Venn diagram analysis, we analyzed the number of contigs that were differentially expressed between multiple tissues (Fig. 2a). Notably, $3982+34+14+95$ contigs were differentially expressed between stem and leaf tissues in both induced and control plants (Fig. 2a). Only 252 contigs were differentially expressed between induced and control stems as well as between leaves and stems in induced plants, but not in induced leaves compared to control leaves, nor in control stems compared to control leaves. This set of 252 contigs represent the set where we hypothesized that genes involved in the production of rubber would most likely be identified. We also examined the number of contigs up- and down-regulated in each comparison (Fig. 2b). More genes were up-regulated than down-regulated in both stem and leaf tissues following cold treatment. Likewise, cold-induction resulted in the up-regulation of only 205 contigs in leaf tissues and the down-regulation of 116 contigs.

This discrepancy between the number of genes differentially expressed between tissue types and the much smaller number differentially expressed between growth

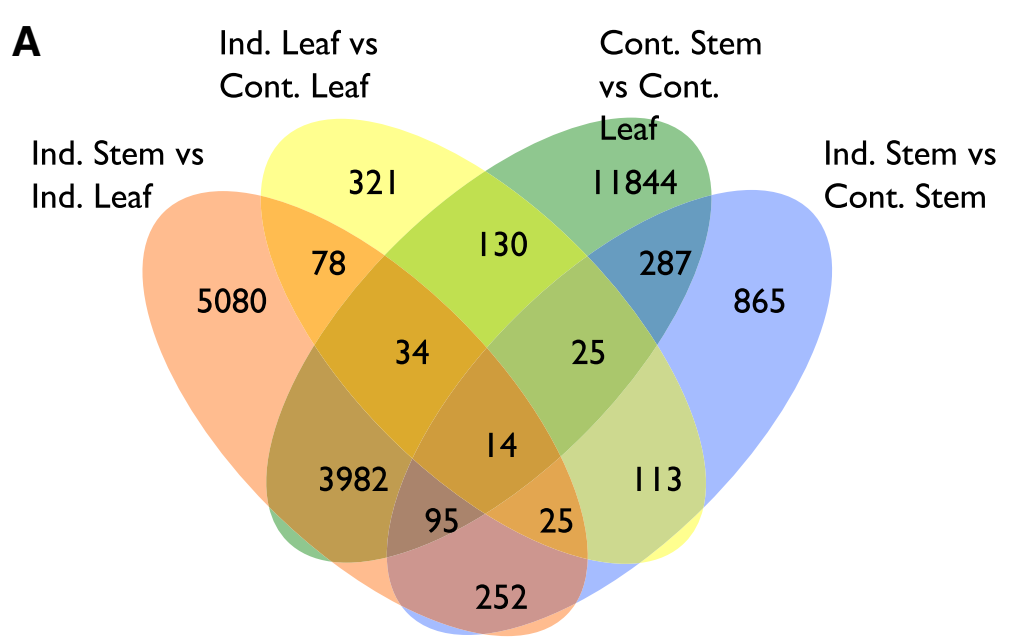

\section{B \# of genes differentially expressed}

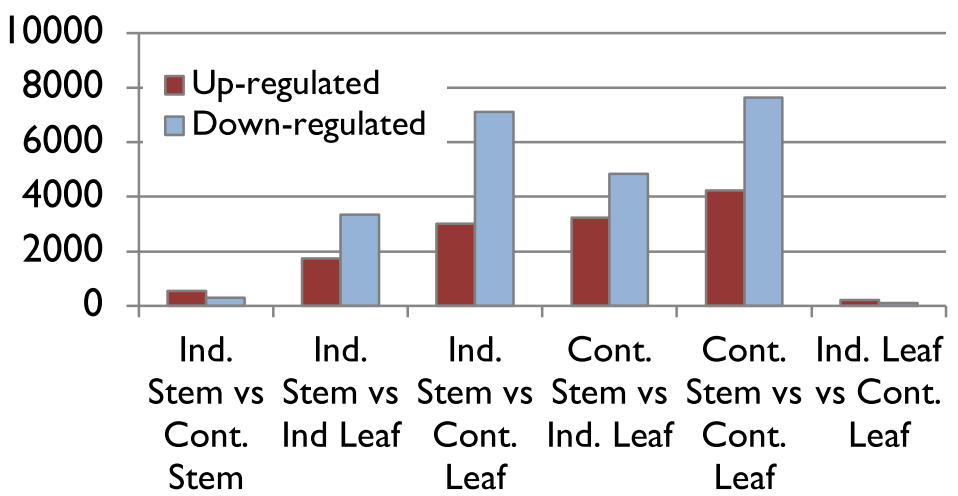

Fig. 2 Differential gene expression in guayule transcriptome analysis. a Venn diagram showing the number of differentially expressed genes in common between different tissues. $\mathbf{b}$ The number of up and down-regulated genes in comparisons of gene expression between each tissue 
conditions is easily observed in a heat map of hierarchically clustered expression data (Fig. 3a). Hierarchical clustering of differentially expressed contigs shows that most genes exhibit similar expression patterns between tissues in both induced and control plants while a smaller group of genes are specifically expressed in the rubberproducing stems of cold-induced plants (Fig. 3a, green bar). Most contigs in this cluster have a background level of expression in the stems of control plants and are more highly expressed in the stems of induced plants.

\section{Gene ontology analysis}

To characterize changes in gene expression between tissues, we analyzed the gene ontology term (GOterm) enrichment of differentially expressed contigs with similar expression patterns marked with colored bars in Fig. 3a. We analyzed the GO-terms enriched in each cluster of differentially expressed contigs. In the cluster of contigs most highly expressed in stems of control plants we observed the enrichment of cytoskeletal, lignin catabolic, and photoreceptor activities at $p$ values below 0.005 (Fig. $3 \mathrm{~b}$ ). In the
A
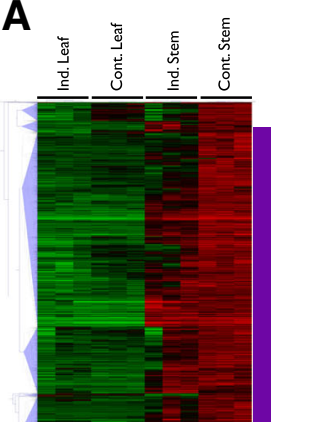

B
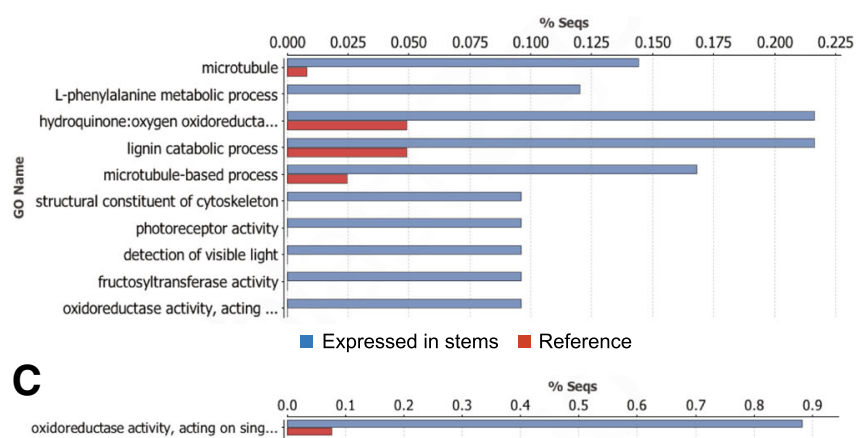

oxidoreductase activity, acting on sing.

chlorophyllide a oxygenase [overal. 2 iron, 2 sulfur duster binding

hydroxymethylglutaryl-CoA reducta...
coenzyme A metabolic process coenzyme A metabolic process

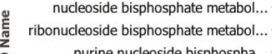
¿ purine nucleoside bisphospha... oxidoreductase activity, acting on sing... carbon-oxygen lyase activity, acting ... terpene synthase activity
iron-suffur duster binding metal cluster binding

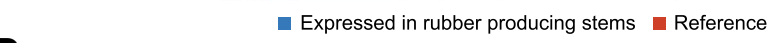

Expressed in rubber producing stems $\quad$ Reference

D

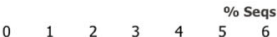

integral component of membrane
chloroplast thylakoid membrane. photosynthesis, light havresting

chophyll binding

chlorophyll binding
protein-chromophore linkage

ATP binding
transferase activity, transferri...

photosystem II oxygen evolvi...

protein phosphonlation.

prase activity, transferri...

quercetin-3-sulfate ...

fatty acid biosynthetic process

photosystem I reaction center

ATP metabolic process
calcium ion binding
carbon fixation

FMN binding $F$

ATPase activity

DNA binding

photorespiration

ribulose-bisphospha...

iron ion binding

electron transport chain.

cell redox homeostasis $F$

electron carrier activity

cation transmembra....

protein binding

protein binding
others

Expressed in leaves Reference

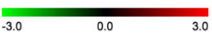

Log2 FC

Fig. 3 Hierarchical clustering and analysis of differentially expressed contigs a) Hierarchical clustering of differentially expressed transcripts. Clusters of contigs with highest expression in specific tissues are highlighted and further examined by GO-term enrichment analysis: stems (purple, b), rubber producing, induced stems (green, c) and leaves (yellow, d). GO terms significantly enriched using in the cluster of contigs most highly expressed in stems $(\mathbf{b})$ at a $P$ value of $<0.005$, in rubber-producing induced stems (c) at a P value $<0.0001$ and in leaves (d) at a P value of $<0.0001$ (Fisher's exact test) 
cluster of contigs highly expressed in the rubber-producing stems of induced plants contigs encoding hydroxymethylglutaryl-CoA reductases, oxidoreductase and terpene synthase activities, which include enzymes families known to be involved in rubber biosynthesis, were enriched at $p$ values below 0.001 (Fig. 3c). Nucleoside metabolic processes were also enriched in the rubber biosynthetic gene set. We also analyzed GO term enrichment in contigs highly expressed in leaf tissues (Fig. 3d). As expected, most of the GO-terms enriched in the leaf-expressed gene set were for processes related to photosynthesis such as chlorophyll binding, ATP metabolic processes and electron transport chain at $\mathrm{p}$ values below 0.001 . Membrane proteins were overrepresented in this gene set, as were enzymatic activities associated with carbon fixation and electron transport chains.

\section{Analysis of highly expressed and strongly induced genes} To elucidate the metabolic processes occurring in rubber biosynthetic tissues, we analyzed the contigs to identify the most highly expressed (Fragments Per Kilobase of transcript per Million mapped reads $($ FPKM $)>256)$ and most strongly enriched $\left(\mathrm{FPKM}_{\text {Induced Stem }} / \mathrm{FPKM}_{\text {Control }}\right.$ Stem $>13$ ) transcripts in induced stem tissues. (Additional file 2: Tables S1 and S2). The most highly-expressed and among the most strongly enriched transcripts in rubber biosynthetic tissue encodes a protein similar to the ribosome-inactivating lectin that is processed into ricin toxin, (TR149020_c3_g1_i1), which is expressed at 7583 FPKM in rubber-producing stems. That this lectin has not previously been identified in proteomic analyses of guayule rubber may be due to the absence of strong homologs in Genbank when previous studies were conducted, or the possibility that this lectin is not bound to guayule rubber particles and is not abundant in preparations of washed rubber particles. Other highly expressed genes are involved in responses to stress and defense responses such as defensins, dehydrins, heat shock proteins (HSPs), metallothionin, COLD-REGULATED 413 and RCI2A. This finding, along with the induction of contigs encoding chitinases implies that the production of rubber in guayule may have a role in defense against herbivory and fungal pathogens as does the production of latex in Hevea. Abundant proteins in Hevea latex have defense roles; hevein, the primary allergen in Hevea latex, constituting $20 \%$ of the dry weight of the lutoid fraction, has chitinase and antifungal activity [18]. Hevein functionally agglutinates rubber particles in Hevea latex and this activity along with its chitinase and chitin-binding activities are thought to function in protection against insects and fungi.

Other highly expressed contigs in rubber-producing tissue encode proteins with roles in defense, including TR8884_c0_g1_i1, encoding a defensin family protein, and two contigs with homology to MLO6 (Mildew Resistance Locus $\mathrm{O}$ 6). Several contigs encoding proteins previously identified as being involved in responses to cold were identified, including contigs encoding homologs of HSPs, COR413-PM2, RCI2A and a dehydrin. A contig encoding a homeobox transcription factor with homology to ATHB7 was also highly expressed. ATHB7 is both cold and drought responsive in Arabidopsis and along with ATHB12 is thought to fine-tune growth during water stress [19].

Analysis of the contigs most strongly induced in stem tissue (Additional file 2: Table S2) identified many additional genes with putative functions in responses to stress and in the production of terpenoids. Numerous HSP-encoding contigs were strongly induced in stems following cold treatment. Several contigs encoding oxygenases were strongly induced, including several with homology to ACD1-like (Pheophorbide-a-oxygenase), a 2-oxoglutarate and Fe(II)-dependent oxygenase-family protein as well as CYP79A2, which catalyzes the monooxygenation of phenylalanine in the biosynthetic pathway for benzylglucosinolate in Arabidopsis [20]. Several strongly induced contigs encode terpene synthase-like enzymes with homology to TPS02 and ATTPS-CIN, which catalyze the production of farnesene from farnesyl diphosphate and diterpenoids from geranyl diphosphate, respectively. The contig TR96894_co_g1_i1 has homology with OLEOSIN1. Oleosins are structural components of oil bodies in plants and affect the size and lipid composition of oil bodies [21, 22], which is relevant given that rubber particles are thought to be structurally related to oil bodies and the small rubber particle protein (SRPP) is homologous to the lipid droplet associated proteins (LDAPs) [23]. Further studies should examine the roles of terpene synthases and oleosin in the biosynthesis of rubber in guayule.

\section{JA biosynthetic genes}

In previous analyses, the most abundant protein found in guayule rubber particles, comprising up to $50 \%$ of total protein was found to be a cytochrome p450 enzyme homologous to Allene Oxide Synthase (AOS) [24], a normally plastid-localized protein catalyzing a reaction in the biosynthetic pathway for jasmonic acid (JA), a terpenoid plant hormone. The role of AOS in rubber biosynthesis remains unclear. One homolog of AOS is highly expressed in rubber-producing tissue (Additional file 2: Table S3) while other homologs have distinct expression patterns with highest expression in leaves. Likewise, distinct orthologs of lipoxygenase and allene oxide cyclase, the enzymes that produce allene oxides, are also upregulated in rubber-producing tissue. Homologs of enzymes beyond the allene oxide cyclase step of JA biosynthesis are not significantly upregulated in rubber 
producing tissue. This result was unexpected and may suggest that AOS has a biochemical role in rubber biosynthesis other than the production of JA.

\section{Identification of rubber biosynthetic enzymes}

To characterize the expression of contigs encoding enzymes previously implicated in the production of rubber in guayule we searched the differentially expressed gene set for contigs encoding the small rubber particle protein (SRPP), rubber associated Cis-prenyltransferases (CPT and CPTL), allene oxide synthase (AOS), DXP and mevalonate pathway enzymes using BLAST, then retrieved annotation and expression data for the identified contigs. Through this analysis we identified a set of contigs encoding AOS, CPTs, SRPP, 3-hydroxy-3-methylglutaryl-coenzyme A reductase (HMGR) and geranyl diphosphate synthase that were generally strongly expressed in induced stem tissue and with lower levels of expression in the stems of control plants (Additional file 2: Table S3A). A consistent background level of expression between one third and one half the absolute transcript level in induced stem tissue was observed in the stems of control plants, in agreement with the qPCR data for selected genes in Additional file 1: Figure S1. The high standard deviation of transcript levels in this study, likely due to the genetic heterogeneity of the polyploid plants sequenced, causes comparisons of expression levels between induced and control stems to be above the stringent threshold used for calling statistical significance. The varying levels of expression of these contigs agree well with qPCR data (Additional file 1: Fig. S1) and previous analyses such as [12]. Each of the contigs identified in this analysis is significantly up-regulated in induced stems compared to expression levels in leaf tissues and is more highly expressed in induced stems than in control stems but not at a significance level of $p<0.0001$. The weak, but consistent induction of the SRPP-encoding gene and the strong expression of AOS in rubber-producing tissue are consistent with previous studies. The presence of many contigs encoding HMGR may also suggest the presence of multiple alleles or tandem duplication and diversification of this gene in guayule and was also observed in GO term enrichment analysis. A previous EST study identified two distinct HMGR encoding ESTs and did not detect cold induction of HMGR [12] although another study [16] supported that the induced rate of HMGR limits the rate of rubber formation in guayule. Other mevalonate and 1-deoxy-D-xylulose 5-phosphate (DXP) isoprenoid biosynthesis pathway enzymes were identified in the set of differentially expressed transcripts, however all were more strongly expressed in leaves than in stems (Additional file 2: Table S3B) and were not significantly up-regulated in stems following cold induction. Homologs of both classes of rubber-biosynthesis-associated CPTs were detected, homologs of Arabidopsis cis-prenyltransferase 1 (CPT1) and Nogo-B receptor-like CPTL/LEW1 [25]. Notably, the latter is strongly implicated in rubber biosynthesis in Russian dandelion, but homologs are absent in the Hevea brasiliensis genome, indicating that the mechanism of rubber biosynthesis may differ between species [26].

\section{Transcription factors expressed in rubber biosynthetic tissues}

To identify transcription factor-encoding genes differentially expressed in rubber biosynthetic tissue we searched for contigs with annotated DNA-binding or transcriptional regulation activity in the GO term dataset. 708 contigs were identified with predicted DNA binding or transcriptional regulation activities. We used hierarchical clustering of the expression of these differentially expressed contigs encoding proteins with DNA-binding or transcriptional regulation activity to identify a cluster with stronger expression in induced stems than in other tissues and at least 2.7-fold higher expression in induced stems than in control stems (Fig. 4, yellow box). This gene set consists of 30 contigs encoding proteins with homology to DNA binding or transcription factors. All but one contig identified through this analysis encode a gene product with homology to previously studied families of transcription factors. TR97361_c0_g6_i1, encodes a homolog of AtGRP2B, which has nucleic acid binding domains and therefore was retrieved in this analysis, but has been shown to act as an RNA chaperone, not a transcription factor [27]. AtGRP2B is a cold-shock domain protein thought to function in the destabilization of RNA secondary structure. Some but not all of these contigs are differentially expressed at $p<0.0001$ between induced stems and all other tissues and many distinct classes of plant transcription factors are represented in the gene set (Additional file 2: Table S4).

\section{Discussion}

Transcriptome analysis of cold-induced guayle stem resulted in identification of several highly induced genes. The experimental approach using leaves and summer simulated plants as controls allowed elimination of induced genes unlikely to be involved in rubber biosynthesis and its regulation. The identified genes included several that are known or expected to be involved in phenylpropanoid and rubber biosynthesis, thus confirming the validity of our approach. Rubber induction was very highly induced in our plants by the cold treatment (Additional file 1: Figure S1), more than would perhaps be expected by the increased transcripts of known or suspected rubber biosynthetic genes (Additional file 1: Figure S1C and Additional file 2: Table S3). However, the pathway for rubber biosynthesis is not fully understood, and the main rate limiting steps could involve 


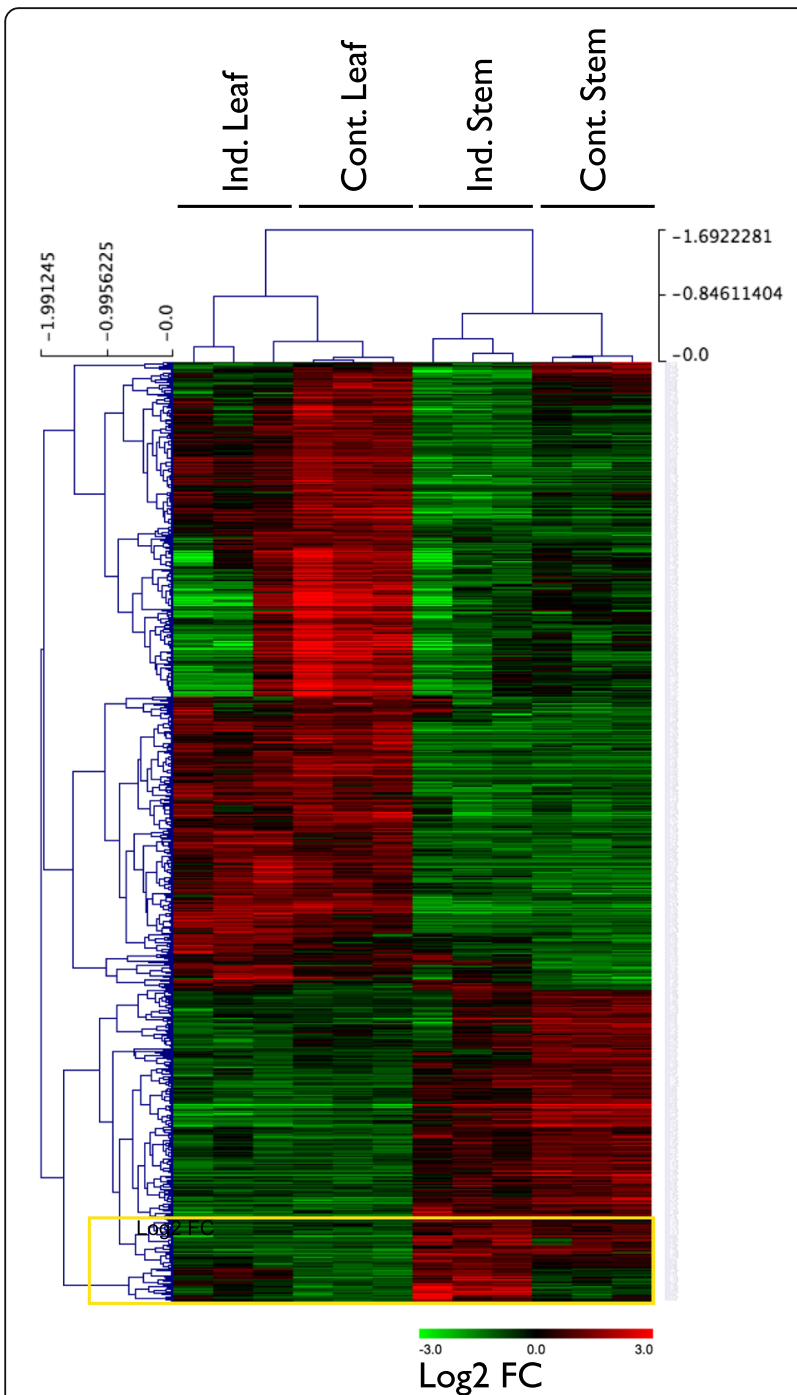

Fig. 4 Clustering analysis of transcripts encoding proteins with homology to DNA-binding proteins or transcription factors. Transcripts clustered together and most highly expressed in induced stems are marked by a yellow box and are further examined in Additional file 2: Table S4

proteins that have not yet been identified. Notably, Additional file 2: Table S2 shows several contigs of proteins with no or limited known functions that highly expressed in induced stems and specifically upregulated in that tissue. These would be good candidates for future studies to validate their possible involvement in rubber biosynthesis. We were particularly interested in transcription factors, since master regulators are ideal targets for genetic engineering of crops. Upregulation of a master regulator will in principle upregulate the entire biosynthetic pathway, and important accessory pathways e.g. for substrate production [28]. Knowing all the biosynthetic enzymes is not necessary for such an approach. In this study we identified several putative guayule transcription factors, which have Arabidopsis homologs with demonstrated roles in the response to abiotic stress or to ABA; ATHB7. DREB1D/CBF4, RD26, AtGSFB2A, AtMYB14, HAT22/ABIG1 AtHSFA2 and AtHSFA6B have been shown to have roles in the response to abiotic stresses or to ABA. These guayule transcription factors are good candidates for regulators of the rubber biosynthetic process given that rubber is produced in a specific tissue, in response to cold. ATHB7 is responsive to drought stress and represses water loss in Arabidopsis [19]. TR42239_c0_g3_i1 and TR42239_c0_g1_i1 are specifically expressed in induced stem tissues and are homologs of DREBA4, a member of the ERF/AP2 family of transcription factors, which are broadly involved in responses to stress and in regulating development. TR78450_c1_g1_i1 and TR78450_c1_g2_i1 encode homologs of DREB1D/CBF4, which is a well-characterized member of the dehydration-responsive element binding and C-repeat binding factor subgroups of ERF/AP2 family transcription factors. Previous studies have shown this subgroup of transcription factors to regulate acclimation to drought and cold stresses [29]. Overexpression of a homolog of CBF4 in poplar induces constitutive tolerance for multiple abiotic stresses including both drought and cold [30]. Thus, overexpression of a CBF4 homolog alone in poplar induced physiological responses associated with acclimation to and conferring tolerance for abiotic stresses. In Arabidopsis, RD26 interacts with BES1/BZR family, brassinosteroid-responsive transcription factors to mediate crosstalk between drought and brassinosteroid signaling [31]. TR91940_c0_g1_11 encodes a homolog of AtMYB14, which mediates cold tolerance in Arabidopsis and was found to negatively regulate freezing tolerance [32]. ABA INSENSITIVE GROWTH (ABIG1) is a stress and $A B A$ regulated transcription factor that functions to restrict growth and promote senescence in Arabidopsis [33]. A guayule homolog of ABIG1 is induced in stems following cold treatment and weakly induced in coldtreated leaves.

\section{Conclusion}

The gene sets identified in this study include strong candidates for master regulators of the cold-responsive, stem-specific biosynthesis of rubber in guayule. Further studies should examine potential uses of these transcription factors for the improvement of rubber yields in guayule.

\section{Methods}

Plant growth

Guayule (Parthenium argentatum Gray) plants of the AZ-2 cultivar were germinated in a greenhouse in Eloy, AZ. At 4 months of age these plants were transferred to simulated summer conditions in a plant growth chamber 
(16 h day, $25^{\circ} \mathrm{C}$ daytime and $15^{\circ} \mathrm{C}$ nighttime temperatures). At 6 months of age plants were transferred to a growth chamber simulating winter conditions $(11 \mathrm{~h}$ night, $25^{\circ} \mathrm{C}$ daytime and $5{ }^{\circ} \mathrm{C}$ nighttime temperatures) for induction of rubber biosynthesis. Plants were harvested for analysis at 8 months of age. Plants grown in simulated winter conditions were considered "induced" for rubber biosynthesis. Washed rubber particle extractions were conducted as described by [34].

\section{Quantitative RT-PCR}

RNA was extracted from plant tissues using Trizol (Invitrogen) following the manufacturer's instructions with the exception that following the initial homogenization and incubation in Trizol, insoluble material was pelleted by centrifugation. Following Trizol extraction, RNA samples were treated with DNAase using the Turbo DNAfree kit (Ambion) and further purified using RNeasy column purification (Qiagen). RNA concentration was measured on a Nanodrop spectrophotometer. RNA was reverse transcribed using the Superscript III kit from Invitrogen. Primer sets for qPCR were designed based on Parthenium argentatum sequences previously deposited in Genbank as well as EST data from the Compositae genomics project (see Additional file 3). qPCR was conducted on a Stepone Real-Time PCR system (Applied Biosystems) using SYBR ${ }^{\circ}$ Select Master Mix (Thermo Fisher Scientific) according to the manufacturer's instructions. qPCR analysis was conducted in biological duplicate with triplicate technical replication.

\section{RNA seq analysis}

Directional, bar-coded illumina sequencing libraries were prepared using the NEBNext ${ }^{\circ}$ Ultra $^{\mathrm{Tn}}$ Directional RNA Library Prep Kit for Illumina ${ }^{\circ}$ (New England Biolabs) according to the manufacturer's instructions with the suggestions for size selection of an average insert size of $300-450 \mathrm{bp}$. For library preparation, RNA samples were prepared as for $\mathrm{qPCR}$ analysis and analyzed using a Bioanalyzer 2100 RNA chip (Agilent Genomics) to evaluate RNA concentration and quality prior to library preparation. Libraries were analyzed on a Bioanalyzer 2100 High Sensitivity DNA assay, pooled at an equimolar ratio. The pooled libraries were then sequenced on Illumina Miseq (2x300bp) and HiSeq2500 (2x150bp, Rapid Run mode) platforms.

\section{Transcriptome analysis}

RNA seq data was quality controlled and adapter sequences removed using Trim Galore (Babraham Bioinformatics group) to eliminate adapter sequence contamination and to trim data below Q30. The command used was 'trim galore --quality 30 --paired --adapter AGATCGGAAGAGCG TCGTGTAGGGAAAGAGTGT --adapter2 AGATCGGAA
GAGCACACGTCTGAACTCCAGTCA --length $30 \quad \$ 1$ $\$ 2$ '. Miseq and Hiseq reads were pooled for transcriptome assembly. The transcriptome was assembled using Trinity assembler version 2.04 set to minimum kmer coverage of 2 [35]. The command used was "Trinity --seqType fq --max_memory 240G --SS_lib_type RF --min_kmer_cov 2 --CPU 40 -left". The initial transcriptome assembly was filtered using a FPKM cut-off of 0.5 to eliminate transcript isoforms with low abundance and low coverage. Differential gene expression analysis was performed using the EdgeR as described in [35]. The differentially expressed gene set was selected as contigs with a $P$ value, adjusted for the false-discovery rate, below 0.0001 .

\section{Functional annotation}

Functional annotation of contigs was performed by searching against NCBI's non-redundant protein sequence database (NR), the Arabidopsis predicted protein sequence database and the Streptophyte predicted protein database retrieved from UniProt, using BLASTx with default parameters. GO functional categories were assigned to differentially expressed genes by homology using Blast2GO (BioBam, Spain). Hierarchical clustering analysis was performed and visualized using Multiple Experiment Viewer version 4.8 [36]. GO-term enrichment analysis was performed and plotted using Fischer's exact test implemented in Blast2GO.

\section{Additional files}

Additional file 1: Figure S1. Images of induced and control plants analyzed in the RNAseq study. (PDF 547 kb)

Additional file 2: Tables S1-S4 in Excel format. Table S1. The most highly expressed contigs in rubber-producing induced stem tissue. Table S2. The most strongly induced contigs in rubber-producing induced stem tissue. Table S3. Contigs encoding enzymes that are implicated in the biosynthesis of rubber and isoprenoids and are strongly expressed in rubber biosynthetic tissue. Table S4. Transcription factor-encoding contigs most highly expressed in rubber-producing stem tissues. (XLSX $33 \mathrm{~kb}$ )

Additional file 3: Table S5. Primer sequences used for RT-PCR (PDF 20 kb)

\section{Acknowledgements}

The authors would like to thank David Dierig at the Bridgestone Americas Inc., Guayule Research Farm in Eloy, AZ for supporting our work and for providing the guayule plants used in this study. Dr. Mi Yeon Lee is thanked for help with plant growth experiments.

This work used the Vincent J. Coates Genomics Sequencing Laboratory and the Computational Genomics Research Lab at UC Berkeley.

\section{Author contributions}

SHS \& HVS designed the research project. SHS generated and analyzed data. SHS \& HVS wrote the manuscript and approved the final manuscript.

\section{Funding}

This work was supported by a cooperative research and development agreement (CRADA10138) between Lawrence Berkeley National Laboratory and Bridgestone Research, LLC.

The work made use of facilities provided by the Joint Bioenergy Institute supported by the U.S. Department of Energy, Office of Science, Office of 
Biological and Environmental Research, through contract DE-AC02-05CH11231 between Lawrence Berkeley National Lab and the U.S. Department of Energy.

\section{Availability of data and materials}

The datasets generated and analyzed in this study are available in the Short Read Archive and the Transcriptome Shotgun Assembly Sequence Database in BioProject PRJNA387289 (https://www.ncbi.nlm.nih.gov/bioproject/ PRJNA387289). The deposited data includes full sequence of all 219,819 contigs. Summarized data are included in this published article and its supplementary information files.

\section{Ethics approval and consent to participate}

Not applicable.

\section{Consent for publication}

Not applicable.

\section{Competing interests}

The authors declare that they have a pending patent application based on the presented data.

\section{Publisher's Note}

Springer Nature remains neutral with regard to jurisdictional claims in published maps and institutional affiliations.

\section{Received: 9 August 2018 Accepted: 31 January 2019}

Published online: 12 February 2019

\section{References}

1. Group IRS. Statistical summary of world rubber situation. http://www. rubberstudy.com/statistics.aspx. Accessed 4 Feb 2019

2. Ahrends A, Hollingsworth PM, Ziegler AD, et al. Current trends of rubbe plantation expansion may threaten biodiversity and livelihoods. Global Environ Chang. 2015:34:48-58.

3. Hamilton RG, Cornish K. Immunogenicity studies of guayule and guayule latex in occupationally exposed workers. Ind Crop Prod. 2010;31:197-201.

4. Kuruvadi S, Rodríguez MEA, López-Benítez A. Guayule ploidy levels in a native population. Ind Crop Prod. 1997:6:83-7.

5. Gore MA, Coyle G, Friebe B, Coffelt TA, Salvucci ME. Complex ploidy level variation in guayule breeding programs. Crop Sci. 2011;51:210.

6. Lloyd FE. Guayule (Parthenium Argentatum gray): a rubber-plant of the Chihuhuan Desert. Washington. In: D.C.: Carnegie Institute; 1911

7. Joseph JP, Shah JJ, Inamdar JA. Distribution, development and structure of resin ducts in guayule (Parthenium argentatum gray). Ann Bot. 1988;61:377-87.

8. Bonner J. Effects of temperature on rubber accumulation by the guayule plant. Bot Gazette. 1943;105:233-43.

9. Downes RW, Tonnet ML. Effect of environmental conditions on growth and rubber production of guayule (Parthenium argentatum). Aust J Agric Res. 1985:36:285-94

10. Veatch-Blohm ME, Ray DT, Gehrels A. Night temperature, rubber production, and carbon exchange in guayule. Ind Crop Prod. 2007;25:34-43.

11. Cornish K, Backhaus RA. Induction of rubber transferase activity in guayule (Parthenium argentatum gray) by low temperatures. Ind Crop Prod. 2003;17: 83-92.

12. Ponciano G, McMahan CM, Xie W, et al. Transcriptome and gene expression analysis in cold-acclimated guayule (Parthenium argentatum) rubberproducing tissue. Phytochemistry. 2012;79:57-66.

13. Ji W, Benedict CR, Foster MA. Seasonal variations in rubber biosynthesis, 3hydroxy-3-methylglutaryl-coenzyme a reductase, and rubber transferase activities in Parthenium argentatum in the Chihuahuan Desert. Plant Physiol. 1993;103:535-42

14. Benedict HM. The effect of soil temperature on guayule plants. Plant Physiol. 1950;25:377-88.

15. Goss RA, Benedict CR, Keithly JH, Nessler CL, Stipanovic RD. cis-Polyisoprene synthesis in guayule plants (Parthenium argentatum gray) exposed to low, nonfreezing temperatures. Plant Physiol. 1984;74:534-7.

16. Benedict CR, Rosenfield CL. W.Ji CL, Foster MA. The low temperature induced rate of 3-hydroxy-3-methylglutaryl-CoA reductase in Parthenium argentatum gray limits the theoretical rate of rubber formation. Ind Crop Prod. 2014;61:176-9
17. Robinson MD, McCarthy DJ, Smyth GK. edgeR: a Bioconductor package for differential expression analysis of digital gene expression data. Bioinformatics. 2010;26:139-40.

18. Berthelot $\mathrm{K}$, Peruch F, Lecomte S. Highlights on Hevea brasiliensis (pro)hevein proteins. Biochimie. 2016;127:258-70.

19. Capella M, Ré DA, Arce AL, Chan RL. Plant homeodomain-leucine zipper I transcription factors exhibit different functional AHA motifs that selectively interact with TBP or/and TFIIB. Plant Cell Rep. 2014;33:955-67.

20. Wittstock U. Cytochrome P450 CYP79A2 from Arabidopsis thaliana L. catalyzes the conversion of L-phenylalanine to Phenylacetaldoxime in the biosynthesis of Benzylglucosinolate. J Biol Chem. 2000;275:14659-66.

21. Siloto RM, Findlay K, Lopez-Villalobos A, Yeung EC, Nykiforuk CL, Moloney MM. The accumulation of oleosins determines the size of seed oilbodies in Arabidopsis. Plant Cell. 2006;18:1961-74.

22. Miquel M, Trigui G, d'Andréa S, et al. Specialization of oleosins in oil body dynamics during seed development in Arabidopsis seeds. Plant Physiol. 2014;164:1866-78.

23. Horn PJ, James CN, Gidda SK, et al. Identification of a new class of lipid droplet-associated proteins in plants. Plant Physiol. 2013;162:1926-36.

24. Pan Z, Durst F, Werck-Reichhart D, et al. The major protein of guayule rubber particles is a cytochrome P450. J Biol Chem. 1995;270:8487-94.

25. Qu Y, Chakrabarty R, Tran HT, et al. A lettuce (Lactuca sativa) homolog of human Nogo-B receptor interacts with cis-prenyltransferase and is necessary for natural rubber biosynthesis. J Biol Chem. 2015:290:1898-914.

26. Tang C, Yang M, Fang Y, Luo Y, Gao S, Xiao X, An Z, Zhou B, Zhang B, Tan $X$, et al. The rubber tree genome reveals new insights into rubber production and species adaptation. Nat Plants. 2016;2(6):16073.

27. Sasaki K, Kim MH, Imai R. Arabidopsis COLD SHOCK DOMAIN PROTEIN2 is a RNA chaperone that is regulated by cold and developmental signals. Biochem Biophys Res Commun. 2007;364:633-8.

28. Zhu Y, Xie L, Chen GQ, Lee MY, Loque D, Scheller HV. A transgene design for enhancing oil content in Arabidopsis and Camelina seeds. Biotechnol Biofuels. 2018:11:46

29. Park S, Lee CM, Doherty CJ, Gilmour SJ, Kim Y, Thomashow MF. Regulation of the Arabidopsis CBF regulon by a complex low-temperature regulatory network. Plant J. 2015;82:193-207.

30. Tian $\mathrm{Q}$, Chen J, Wang $\mathrm{D}$, et al. Overexpression of a Populus euphratica CBF4 gene in poplar confers tolerance to multiple stresses. Plant Cell Tissue Organ Cult. 2017;128:391-407.

31. Ye H, Liu S, Tang B, et al. RD26 mediates crosstalk between drought and brassinosteroid signalling pathways. Nat Commun. 2017;8:14573.

32. Chen Y, Chen Z, Kang J, Kang D, Gu H, Qin G. AtMYB14 regulates cold tolerance in Arabidopsis. Plant Mol Biol Report. 2013;31:87-97.

33. Liu T, Longhurst AD, Talavera-Rauh F, Hokin SA, Barton MK. The Arabidopsis transcription factor $A B I G 1$ relays $A B A$ signaled growth inhibition and drought induced senescence. elife. 2016:5.

34. Cornish K, Backhaus RA. Rubber transferase activity in rubber particles of guayule. Phytochemistry. 1990:29:3809-13.

35. Haas BJ, Papanicolaou A, Yassour M, et al. De novo transcript sequence reconstruction from RNA-seq using the trinity platform for reference generation and analysis. Nat Protoc. 2013;8:1494-512

36. Howe EA, Sinha R, Schlauch D, Quackenbush J. RNA-Seq analysis in MeV. Bioinformatics. 2011:27:3209-10.

Ready to submit your research? Choose BMC and benefit from:

- fast, convenient online submission

- thorough peer review by experienced researchers in your field

- rapid publication on acceptance

- support for research data, including large and complex data types

- gold Open Access which fosters wider collaboration and increased citations

- maximum visibility for your research: over $100 \mathrm{M}$ website views per year

At BMC, research is always in progress.

Learn more biomedcentral.com/submissions 